\title{
Research of Key Technologies of Agricultural Products Traceability System
}

\author{
Fang Liu, Lian Xue \\ Information Engineering College,Wuhan Technology and Business University, WuHan 430063, \\ China; \\ liufangteacher1982@126.com
}

Keywords: RFID, agricultural product traceability, GPRS, agricultural sensor

\begin{abstract}
With the application of RFID technology in the Internet of things in agriculture, realize the agricultural products traceability safety, mainly to realize the design of Agricultural Internet of things sensing layer, network layer, application layer. Through RFID label record collection of agricultural products in planting, processing, packaging, warehousing, logistics, sales and other information, Through the reader reads the tag information and transmits them to the agricultural products traceability database, consumers scan two-dimensional code through a variety of mobile terminal, to realize traceability of agricultural products supply chain security.
\end{abstract}

\section{Introduction}

The whole process from the production to the consumption of agricultural products, including cultivation, detection, processing, packaging, transportation, storage, and consumption and other links, each link has the potential to bring unsafe factors. An agricultural product traceability program is necessary, which is the whole process of agricultural products supply chain monitoring, to ensure the quality and safety of agricultural products ${ }^{[1]}$. With the application of RFID technology in the traceability of agricultural products, when the electronic label fitted with information of agricultural products into the reader device antenna field, reader can be in accordance with the provisions of the read write protocol and encryption to read the label on the planting, processing, warehousing, logistics, and other aspects of the information and upload the information to the agricultural products traceability server and updates to the database, also the electronic label received from the server data write request of agricultural products current processing and warehousing logistics information will be written to the tag. The electronic label is pasted on the food, the reader is connected with the antenna, and the sensor is integrated with the sensor. Read data packets is transmited to the food safety management database through the network, and each link in the food supply chain information is registered to RFID public service system. This system provides information service for food safety traceability through the integration of food safety database management and supply chain information ${ }^{[2]}$.

\section{Agricultural products traceability platform solutions}

\subsection{System to achieve the service function}

Based on the integration of agricultural product safety management database and agricultural products supply chain information, the system can provide the following services. agricultural product information service: the user can query the information service of agricultural products. Such as raw material origin, production (processing), production date and other information, supermarket shopping, etc. safety traceability of agricultural products: once the outbreak of the epidemic, through the agricultural product safety management system can quickly find the product sales, responsible person and product origin and other information, to take appropriate measures. terminal inquiry system: the supermarket as the main channel for consumers to buy food, consumers can check the information through the RFID electronic tag terminal, provided by the supermarket $^{[3]}$. 
Tracing system of agricultural products chain mainly includes two parts, a part is collecting the basic information of agricultural products, such as planting information,logistics and supply chain information processing, packaging and sales information, which are acquisited through the RFID radio frequency automatic, transmit information to the database and cloud platform by GPRS or Internet. another part is the consumers get information for traceability of agricultural products by scan the bar code or scan the label, through the web client or Android interface login ${ }^{[4]}$. Supervision part can be traced back to the existence of the problem of the link. Agricultural products traceability solutions rely mainly on each link of intelligent node information, use of electronic tag technology, GPS positioning technology, Internet technology, wireless sensor technology and database technology, uses the intelligent transaction, each node organic unifies in together, is connected to the line sensor network, all data are collected ${ }^{[5]}$, using two-dimensional code, two-dimensional code generation, complete traceability of agricultural products information by mobile phone to scan. The key technologies of Agricultural Internet of things are divided into three levels: perception layer, network layer and application layer. The overall design of the system is shown in Figure 1.

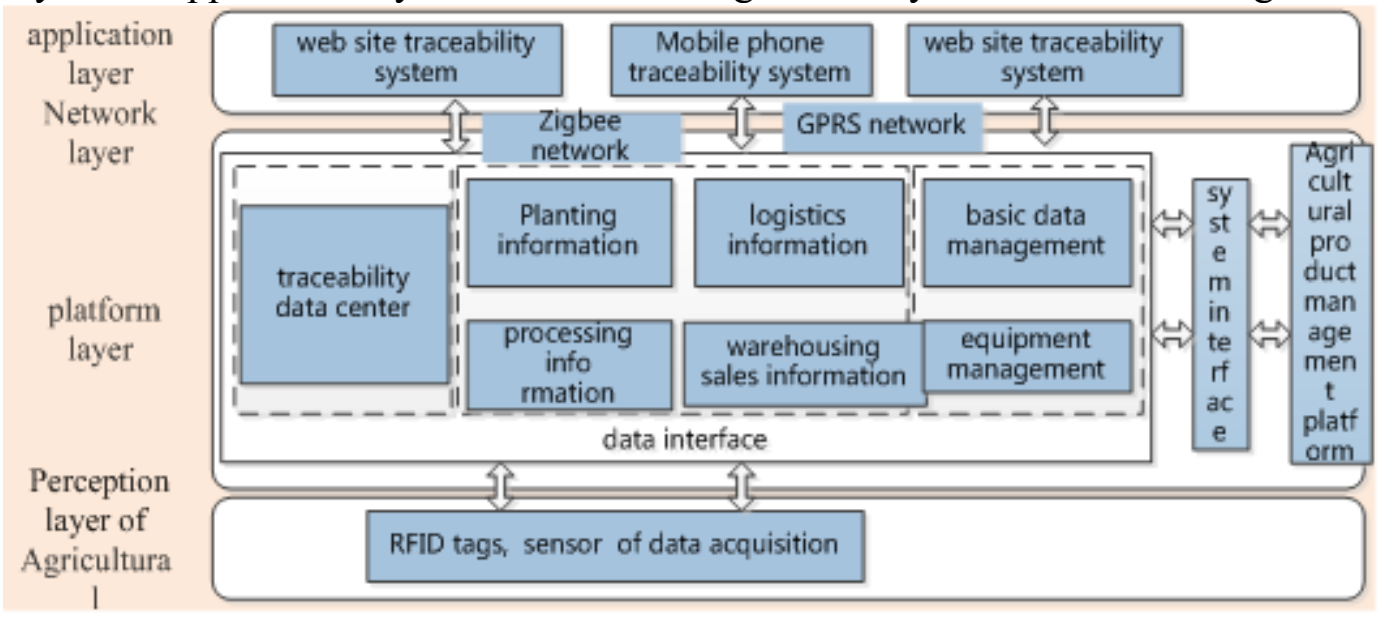

Figure 1 The overall design of the system

\subsection{The Perception layer of Agricultural information}

Agricultural information perception layer includes smart card, RFID tag, identification code and two-dimensional code and read and write device, camera, sensor networks and sensor gateway, which mainly solves the key problem is perception, object recognition, collect the information in this layer ${ }^{[6]}$. Frequency Identification Radio is a radio frequency identification technology, it is a technology that uses radio frequency signal to realize non-contact information transmission through space electromagnetic coupling and to achieve object recognition through the information transmitted.

The electronic label is configured from the agricultural production, processing, logistics to the sales stage through RFID technology and the label with EEPROM and a unique ID number, it can label each kind of agricultural products information, unified global goods coding techniques were used to determine the unique ID number for each food through RFID read or write information on food process, the food chain all the information timely release to the network management platform through the wireless transmission technology at the same time ${ }^{[7]}$. Tracking and tracing of agricultural products and related information, to achieve the effective monitoring of the entire agricultural product supply chain. RFID system is mainly composed of the electronic tag (Tag), tag reader, antenna and database module, figure 2 is a schematic diagram of the structure of a RFID module. 


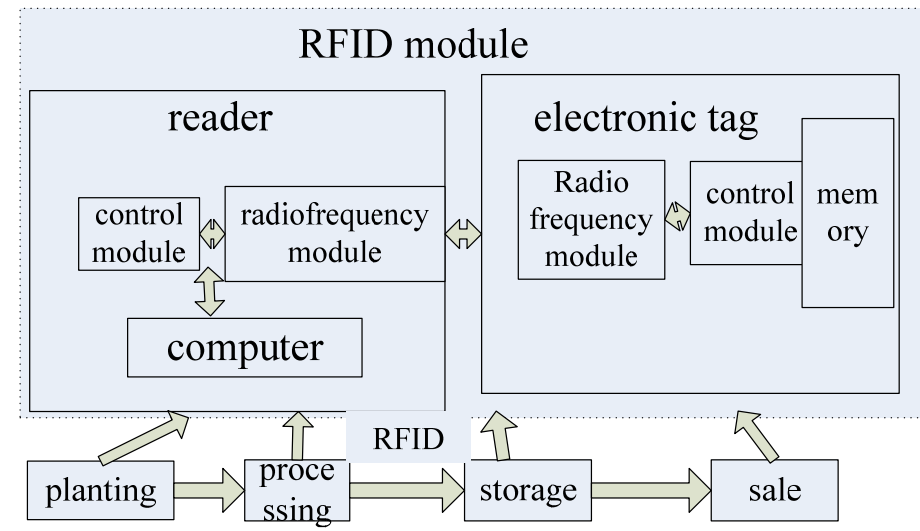

Figure 2 RFID module

\subsection{The network layer of Agricultural information}

The network layer is the bridge of the sensing layer and application layer, which realizes the wireless transmission of agricultural product information through the form of wireless transmission and networking ${ }^{[8]}$. This design uses the Zigbee wireless sensor network, which have the features of ad hoc network, RFID and Zigee module effective combination to achieve each node data transmission; GPRS technology to achieve long-distance communication, which is mainly responsible for transmission and processing from the perception layer and RFID acquisition of agricultural products information, to achieve traceability information data sharing.

2.4 The application layer of Agricultural information

Each agricultural product enterprise has a unique fixed network IP address Web by server technology, Internet users and other ordinary users can access through the network into the food processing enterprises in the local area network ${ }^{[9]}$. In the enterprise management platform to establish a Web server to achieve data remote access, data center using the browser / server $(\mathrm{B} / \mathrm{S})$ mode of operation, the user can view the data through the browser.

\section{The implementation of the platform}

Agricultural product traceability system platform development is based on the JAVA and MySQL database, based on the development of the three tier architecture of the $\mathrm{B} / \mathrm{S}$ model, JAVA language is the foundation of the flexible use of language skills. Implement system of data business logic layer and data access layer using JDBC +SPRING framework, data table maintenance in the business layer and data layer interaction is created according to the need.

Database requires a strong processing capacity, and can be easy to maintain, with good security, in line with the standard、open. Database system should support multi process, multi thread, support Client/Server and SMP processing mode, can support multiple concurrent users, the database has a good query and running performance is also equipped with requirements. In order to improve the query speed, the data is stored in a distributed storage strategy. Traceability model is implemented in the platform, through the link to a specific URL for the query after users scan bar code after the bar.

\section{Agricultural products traceability query results}

Agricultural products terminal traceability system is the information carrier based on the bar code technology and RFID technology, database technology and network technology as a service platform, to achieve a variety of terminal, such as traceability website, bar code scanning of various forms of query. The development of this system is based on the development of the platform, through the query to the source code or scan the corresponding bar code, or query the establishment of the source site, showing the basic information of agricultural products. The design combined with the input information from the structure of the agricultural products supply chain, in the supply 
chain information respectively, showing the corresponding date, such as cultivation, detection, processing, packaging, transportation, storage, and consumption and other links information and detection structure, query results as shown in Figure 3.

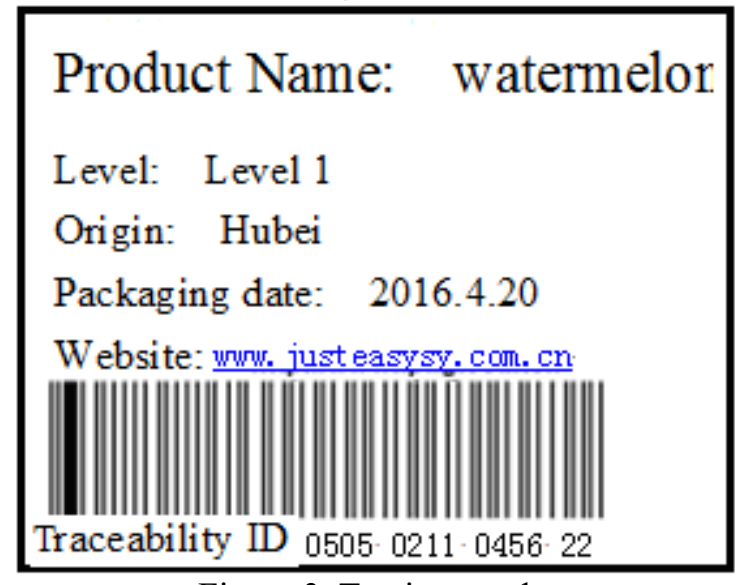

Figure 3. Tracing results

\section{Acknowledgement}

This study greatly thanks to the support of the Department of Internet of things project of Wuhan Technology and Business University, thanks to the research project from Hubei Provincial Department of Education of Science and technology. The Item number is B2015330. Project Name: The key technology research of agricultural product safety traceability system.

\section{Reference}

[1] Luo Aimin, Yi Bin, Shen Caihong. Design and application of food digital ID card based on radio frequency identification [J]. Journal of agricultural engineering, 2013,10 (31): 46-48.

[2] Chen Qingang, Ma space selective color centers formation by the irradiation of near and Li Fengxia, Chen Weidong, Yang Jianchun. Based on RFID and animal products quality safety traceability system [J]. Agricultural Mechanization Research. 2013 (08)

[3] Ma Hongjian, Liu Liu. Design and implementation of traceability system for vegetable supply chain [J]. Journal of agricultural machinery in China, 2015,6 (36): 230-235.

[4] Zhao Jun, Liu Zejun. Development of intelligent parking management system based on [J]. Intelligent computer and application RFSN, 2012,2 (4):86-89.

[5] Pu moonlight, Hai Hui Zhang. Based on RFID technology in small and medium enterprises vegetable traceability system design [J]. Agricultural Mechanization Research, 2015,4, (12) [J].: 207-212.

[6] ZhaoJun,LiuZejun. The Development of Management System of Intelligent Parking based on RFSN.INTELLIGENT COMPUTER AND APPLICATIONS, 2012,2(4): 86-89(in Chinese).

[7] Yang Jiandong, Wang Pu, Sinong. RFID technology quality traceability management system research based on [J]. control engineering, 2015.7 (22): 35-38.

[8] Xia Jun, Ling Peiliang. Products of the whole industry chain network traceability system research and implementation [J]. Journal of Shanghai Ocean University. 2014 (24):304-310.

[9] Liu Yanfei, $\mathrm{Yu} \mathrm{Yu.} \mathrm{Research} \mathrm{on} \mathrm{anti} \mathrm{counterfeiting} \mathrm{system} \mathrm{based} \mathrm{on} \mathrm{Internet} \mathrm{of} \mathrm{things}$ technology, modern electronic technology.2016,3 (10): 34-36. 